\title{
A13
}

doi: 10.14232/ syrpharmacognosy.2021.a13

\section{Cytotoxic and antiproliferative activity of sesquiterpene lactones isolated from the aerial parts of Ambrosia artemisiifolia}

\author{
Balázs Kovács
}

Email: kovacs.balazs@pharmacognosy.hu

Ambrosia artemisiifolia (common ragweed) is an invasive weed in Europe, well-known for the strong allergenic effect of its pollen, as well as for its invasiveness and impact in crop fields. This species produces a broad range of sesquiterpenoids. These bioactive molecules possess allergic, allelophatic [1], anti-inflammatory [2], antitumor [3] and antimicrobial activities [4]. From a phytochemical view the secondary metabolites of the plant have not explored completely. The primary aim of our work was to isolate the major phytochemicals from the aerial parts of $A$. artemisiifolia, focusing on the sesquiterpene lactone content and to elucidate their chemical structure. Subsequently, we performed cytotoxic, antiproliferative and combination assays with the isolated compounds. Also the genus Ambrosia was reviewed focusing especially on the sesquiterpene lactone compounds and their pharmacological effects.

The methanolic extract of the plant was separated by several chromatographic techniques, including preparative TLC and HPLC. Eight compounds were isolated. Their structure was identified by ${ }^{1} \mathrm{H} N M R,{ }^{13} \mathrm{C}$ NMR and mass spectrometry. Two new seco-pseudogauianolides $1^{\prime}$ noraltamisin and 1,10-dihydro-1'-noraltamisin were reported for the first time from this plant. Their cytotoxic and antiproliferative effect were measured on different human cancer cell lines and also on a normal cell line. A combination assay was applied to study the effect of drug interactions between the compounds and doxorubicin.

Supervisor: Csupor Dezső

\section{Acknowledgements:}

We wish to thank Dr. Gabriella Spengler and her colleague Nikoletta Szemerédi for the biological activity assays.

\section{References}

[1] Molinaro F, et al. Journal of Environmental Science and Health 2016;5(1):847-852.

[2] Pérez G. RM Phytomedicine 1996;3: 163-167.

[3] Solujic S, et al. J Serb Chem Soc 2008;73: 1039-1049.

[4] Sturgeon CM, et al. Planta Med 2005;71: 938-943. 